\title{
Dinámicas locales en la implementación del Programa 3x1 para Migrantes: entre el conflicto y la cooperación
}

\author{
Judith Pérez-Soria
}

El Colegio Mexiquense A.C.

\section{Resumen}

El Programa 3x1 para Migrantes es la forma institucional que ha tomado el envío de remesas colectivas a las localidades mexicanas. Desde 2002 es un programa federal, aunque sus antecedentes se encuentran en las organizaciones civiles de inmigrantes y en la permanencia de vínculos sociales con la población de las localidades de origen. Las características del Programa, y la activa participación de los migrantes que presupone, han hecho que sea un tema importante en los estudios de migración internacional y en los estudios de políticas públicas. Desde ambas perspectivas se da cuenta de la heterogeneidad de la implementación del Programa a nivel local, sin embargo son campos de investigación separados. Este estudio es un esfuerzo por complementar los hallazgos en la materia y analizar, a partir de datos cualitativos, dos problemas reportados constantemente: la falta de focalización en zonas de alta marginación y la centralidad de otros actores que no son los migrantes organizados. Los resultados muestran que los intereses, y las diferentes definiciones del problema público que el Programa busca atender, imposibilitan dicha focalización, mientras que la centralidad de otros actores produce efectos opuestos en la rendición de cuentas, dependiendo del actor que sustituya al club de migrantes.

Palabras clave: Programa 3x1 para Migrantes, clubes de migrantes mexicanos, políticas públicas.

Local dynamics in the implementation the Programa 3x1 para Migrantes: between conflict and cooperation

\begin{abstract}
The Programa 3x1 para Migrantes is the institutional shape adopted by the collective remittances sent to Mexican communities. Since 2002 it is a federal governmental program, although it is rooted in Mexican immigrant associations and links between immigrants and local population in Mexico. The Program's main features, as well as the high level of civic participation of immigrant associations it is built upon, make it a key subject in migration studies and public policy studies. Although both approaches find substantial heterogeneity in the Program's implementation at the local level, they remain as different research fields. This article is an effort to complement the different findings in the subject by means of the qualitative analysis of two problems frequently reported: lack of targeting in high-marginalization areas, and the centrality of local actors other than migrant associations. Our findings are twofold. First, it is shown that the differences of interests across actors, and the different definitions of the public problem that the Program displays over time, make the beneficiary areas selected by immigrants incompatible with the government's priorities. Second, we find opposite effects in accountability, depending on the actor that replace the immigrant club as the central actor in the implementation process.
\end{abstract}

Keywords: Programa 3x1 para Migrantes, Mexican hometown associations, public policy.

E-mail: jperez@cmq.edu.mx 


\section{Introducción}

A partir de la década de los noventa se intensificaron en México los programas públicos y las reformas constitucionales para atender a los migrantes mexicanos que se encontraban en el exterior ${ }^{1}$, entre estas acciones se creó el Grupo Beta (1990)², se reformó el artículo 32 sobre el derecho a votar en el extranjero (1996), el artículo 37 para asegurar la no pérdida de la nacionalidad mexicana (1998), se creó del Instituto de los Mexicanos en el Exterior (2002), el Programa Iniciativa Ciudadana 3x1 (2002), el Programa de Repatriación (2007) y la Estrategia Somos Mexicanos (2014).

Por las características de estos programas se habla de una política del reconocimiento (Délano, 2006), porque el gobierno federal mexicano tomó un papel más activo con respecto a la población migrante, a partir de "reconocer" la importancia de la migración internacional y de las remesas enviadas al país. De manera que, por un lado, se diseñaron programas para garantizar un mínimo de derechos a los migrantes y, por el otro, para potencializar las remesas que llegaban al país ${ }^{3}$.

Como parte de estas acciones se implementó el Programa Iniciativa Ciudadana 3x1, en 2002, que consiste en el cofinanciamiento de los tres órdenes de gobierno (federal, estatal y municipal) y del club de migrantes mexicanos, para algún proyecto de infraestructura, servicios básicos o educación en las localidades de México. El objetivo del programa en ese momento era "apoyar las iniciativas ciudadanas para concretar proyectos para mejorar la calidad de vida de los habitantes (...)" (González Rodríguez, 2011: 39$)^{4}$.

El Programa se incluyó dentro de la cartera de programas federales a partir de las negociaciones entre un grupo de migrantes, procedentes de Zacatecas, y las autoridades gubernamentales federales. Sin embargo, esta forma de gestión e inversión en las localidades de origen de los migrantes tiene sus

\footnotetext{
${ }^{1}$ Aunque a finales de los ochenta se implementó el Programa Paisano (1989) y el Programa para las Comunidades de Mexicanos en el Exterior (PCME, 1989 -1990), es a partir de 1990 que se encuentra la mayoría de los programas y reformas constitucionales.

${ }^{2}$ Grupos que brindan orientación, rescate y primeros auxilios a migrantes que se encuentran en el territorio mexicano. Comenzó como un programa piloto en Tijuana, Baja California, y se extendió a nueve estados del país (Baja California, Sonora, Chihuahua, Coahuila, Tamaulipas, Veracruz, Tabasco, Chiapas y Oaxaca).

${ }^{3}$ Como se puede observar no se trata de políticas para detener los flujos migratorios, o política de retención como la denomina Délano (2006), sino de programas para potencializar los efectos económicos de la migración.

${ }^{4}$ Para una revisión de la historia del Programa véase Sánchez y Jiménez (2011) y García Zamora y Padilla (2012).
}

antecedentes en la década de los sesenta (Moctezuma y Pérez Veyna, 2006; Sánchez y Jiménez, 2011).

En esta década el financiamiento, la gestión y la ejecución del proyecto estaban a cargo de los migrantes organizados, en coordinación con algunos habitantes de la localidad de origen, de manera que se realizaba con total independencia de las autoridades gubernamentales. Posteriormente, se fueron sumando los diferentes órdenes de gobierno, por ello, desde un enfoque de políticas públicas se define como un diseño bottom-up, es decir construido desde la sociedad civil organizada, por lo menos para el caso zacatecano.

En 2005 cambió de nombre a "Programa 3x1 para Migrantes", mismo que mantiene hasta la actualidad. Los proyectos financiados en su mayoría son sociales (sobre todo de infraestructura y servicios básicos), en 2009 el mayor porcentaje de proyectos fue de urbanización de plazas, jardines y banquetas $(21.6 \%)$, dotación de agua potable $(21.4 \%)$, pavimentación de calles (11.6\%) y construcción de centros comunitarios (casas de ancianos o bibliotecas) (11.6\%) (Rodríguez Ramírez, 2012). En 2014, del total de proyectos aprobados, 1,527 fueron sociales y 219 productivos (CONEVAL, 2015).

Por sus antecedentes y diseño, que incluye la activa participación de la sociedad civil migrante, el Programa 3x1 ha sido estudiado desde diferentes enfoques teóricos, tales como gobernanza (Burgess, 2006; González Hernández y González Hernández, 2013; Leal Jiménez, 2008), transnacionalismo (Moctezuma y Pérez Veyna, 2006; Shannon, 2006; García Zamora y Padilla, 2012; Faret, 2004; Lanly y Hamann, 2004) y políticas públicas (García Zamora, 2007b; González Ortiz y Rivera, 2004; Rodríguez Ramírez, 2012). Los métodos empleados también son diversos, se utilizan tanto cuantitativos como cualitativos, aunque predominan estos últimos.

Para los propósitos de este artículo se exploran, a partir de entrevistas a integrantes de clubes de migrantes que han participado en el Programa, dos aspectos señalados frecuentemente como problemas para el logro de los objetivos del Programa 3x1 para Migrantes: 1) la baja aplicación en localidades de alta marginación (entendido como un problema de focalización o de indefinición de la población objetivo) y 2) la centralidad de otros actores que no son los migrantes organizados como estipulan las Reglas de Operación (ROP). El estudio parte de que las experiencias de los actores son fundamentales para entender los problemas de diseño e implementación de cualquier política pública.

Para términos de exposición, el artículo se divide 
en tres secciones, más las reflexiones finales. En la primera parte, se presenta la revisión de los estudios sobre el Programa para mostrar convergencias y divergencias en los hallazgos empíricos. Posteriormente, se describe brevemente la metodología del estudio. Y, consecutivamente, se exponen los resultados empíricos sobre: a) la no coincidencia entre población atendida y población objetivo del Programa y b) las implicaciones de la centralidad de presidentes municipales y de la población local en la implementación del mismo.

\section{Aportes separados, hallazgos consistentes}

En la revisión de la literatura sobre el Programa 3x1 para Migrantes se encuentran: investigaciones globales o generales (Raccanello y López Velázquez, 2008; Ruiz-Ochoa, 2009; Vega Godínez y Brindis Almazán, 2011; García Zamora y Padilla, 2012; Rodríguez Ramírez, 2012; Mesenguer y Aparicio, 2012), evaluaciones oficiales (Red de Consultores Nacionales, 2004; SERPRO, 2005, 2006; Universidad Autónoma de Chapingo, 2006; CIDE, 2008, CONEVAL, 2008, 2010, 2011; Ahumada Lobo y Asociados, 2016), y estudios de caso a nivel estatal, municipal y/o local (Burgess, 2006; González Hernández y González Hernández, 2013; García Zamora, 2007b; Ramírez et al., 2011; Valenzuela, 2007; Díaz Garay, 2009; González Ortiz y Rivera, 2004; Vega Briones, 2004; Baca Tavira, 2009; Frías et al., 2007). A continuación se exponen los principales resultados.

\section{Estudios generales sobre el Programa $3 \times 1$ para Migrantes}

En este grupo de estudios se clasificaron investigaciones que analizan el Programa en su conjunto y su efecto sobre alguna dimensión (social, política o económica). La mayoría de estos trabajos utilizan datos cuantitativos, sea que fueron generados ex profeso para la investigación o que se tomaron de las evaluaciones oficiales y de los reportes de la Secretaría de Desarrollo Social (SEDESOL).

Por ejemplo, Raccanello y López Velázquez (2008) encuentran que el porcentaje de residentes en los Estados Unidos, el grado promedio de escolaridad y la población analfabeta de 15 años y más, se relacionan positivamente con la implementación del Programa 3x1 en las localidades mexicanas. Además, reportan que las localidades con niveles de marginación alta y muy alta tienden a no ser beneficiadas, porque los niveles de intensidad migratoria alto y muy alto se encuentran asociados con un nivel de marginación medio.

Por su parte, Ruiz-Ochoa (2009), con los datos de las evaluaciones del Programa de 2002 a 2007, muestra una disminución en los recursos per cápita, en promedio, que recibieron los beneficiarios (de 29 a 15.5 dólares), y considera que el Programa tiene serias limitaciones para detonar efectos multiplicativos sostenibles en el tiempo, porque las inversiones y los proyectos son de montos pequeños y difícilmente pueden impactar a las comunidades con rezagos profundos.

Vega Godínez y Brindis Almazán (2011), por su parte, al estudiar el efecto del Programa en la imagen pública de los migrantes encuentran que, en términos de percepción social, hubo un cambio a partir de inversión de remesas colectivas en las localidades mexicanas, es decir que a partir de la implementación del Programa 3x1, los migrantes son vistos como agentes potenciales del desarrollo local. Así mismo, García Zamora y Padilla (2012) concluyen que el impacto económico, social y cultural del Programa es positivo, y que su éxito se debe a la solidaridad de las organizaciones de migrantes y a las negociaciones transnacionales entre migrantes, comunidades y gobierno.

Mientras que Rodríguez Ramírez (2012) afirma que el Programa es un mecanismo novedoso de participación de los migrantes en las políticas públicas binacionales, porque redefine la colaboración entre actores gubernamentales y no gubernamentales, y crea una agenda "integral" de políticas públicas. Finalmente, Meseguer y Aparicio (2012) analizan las localidades beneficiarias a partir de la competencia electoral municipal y muestran que, en contextos de competencia electoral efectiva, el índice de recursos para proveer bienes públicos, bajo el Programa 3x1, es menor que en jurisdicciones menos competentes (con fuerte presencia de un partido y con un índice de alto de migración). Esto sugiere, según los autores, que hay recompensas políticas debajo de las decisiones de asignación del Programa. Por tanto, se usa como instrumento de intercambio político.

\section{Evaluaciones oficiales del Programa $3 \times 1$ para Migrantes}

En 2002, cuando el Programa se implementó a nivel federal, se realizó la primera evaluación, desde esta fecha y hasta la actualidad, las evaluaciones se han realizado, más o menos, de manera constante. Sin embargo, en los primeros ejercicios se señalaban un diseño correcto para alcanzar los objetivos del Programa (Red de Consultores Nacionales, 2004; SERPRO, 2005, 2006). Incluso en la evaluación de 
2005 se concluye que: "el Programa se encuentra bien focalizado ya que sus recursos y acciones se encuentran canalizados principalmente a zonas de pobreza y alta migración" (SERPRO, 2006: 119).

La evaluación de 2006 es la que planteó la falta de coincidencia entre las localidades beneficiadas y la población objetivo, porque los migrantes elegían las localidades a apoyar, independientemente de los niveles de marginación y migración. En ese año, el Programa había beneficiado, en mayor medida, a la población que habitaba en municipios de baja y muy baja marginación (66 por ciento del total nacional). Y los recursos invertidos se concentraban en cuatro entidades: Zacatecas (27.4 por ciento), Jalisco (33.0 por ciento), Guanajuato (13.2 por ciento) y Michoacán (9.1 por ciento) (Universidad Autónoma de Chapingo, 2006).

Posteriormente, la falta de focalización del Programa en localidades de alta y muy alta marginación continuó siendo señalada como un problema. Por ejemplo, en 2007, se concluyó que los resultados del Programa eran modestos en la atención de las zonas marginadas, porque se partía de un supuesto inválido al considerar que "las zonas de alta migración son también las de alta marginación y rezago social, o bien, que las localidades seleccionadas por los migrantes serán de alta marginación" (CIDE, 2008: 111).

Sobre las evaluaciones posteriores solo se encuentran los documentos emitidos por el Consejo Nacional de Evaluación de la Política de Desarrollo Social (CONEVAL), en donde se señala que el Programa debe atender no sólo a las localidades con altos índices de intensidad migratoria, sino con altos y muy altos niveles de marginación. Además que debe buscar mecanismo para incentivar la participación de municipios y localidades de estados diferentes a Michoacán, Jalisco y Zacatecas (CONEVAL, 2008).

Para 2009, se consideró que la población potencial y objetivo estaban claramente definidas, pero sobredimensionadas con respecto a la población atendida, porque el Programa se basaba en las propuestas de los clubes de migrantes. En ese año se eliminó la prioridad de atender localidades en condiciones de pobreza y alta marginación de las Reglas de Operación, para ajustar fin, propósito y población objetivo (CONEVAL, 2010).

En la valoración que el CONEVAL hizo de la evaluación de 2010-2011 se destaca el incremento del número de clubes y de la inversión de los migrantes en los proyectos. En cuanto a la población objetivo, se considera que está en proceso de definición, porque no es posible conocerla a priori, ya que depende de la población migrante, y se surgiere: reforzar la difusión del Programa, consolidar la participación de los clubes espejo y continuar con la precisión de la población potencial y objetivo (CONEVAL, 2011).

Finalmente, en la Evaluación Complementaria (2013-2014), se reporta el aumento de la percepción sobre la corrupción y la falta de transparencia en los procesos de implementación, así como reducción del interés de los actores en participar en el Programa. Además de una serie de irregularidades en la selección del proyecto y de la localidad beneficiada, porque, en ocasiones, no eran los migrantes los que toman estas decisiones, sino el gobierno municipal, la población de la localidad o alguna dependencia gubernamental estatal (Ahumada Lobo y Asociados, 2016).

Aunque la agencia consultora responsable de esta evaluación afirma que la centralidad de otros actores, que no eran los migrantes, en la ejecución del Programa no estaba considerada, sino que fue "descubierta" en campo, lo cierto en que los estudios cualitativos ya habían reportado diferentes formas de negociación local y tensiones con el presidente municipal. Esta evaluación, considera que la heterogeneidad en la implementación del Programa se origina en "la laxitud y generalidad del marco normativo" (Ahumada Lobo y Asociados, 2016: 207). En cuanto a la cobertura, sugiere que se reduzca hacia localidades y actores que cumplen con la normatividad.

Como se ha mostrado, los estudios generales y las evaluaciones oficiales señalan la falta de focalización en zonas de alta y muy alta marginación como un problema porque reduce la consecución de los objetivos del Programa. Además, de la heterogeneidad en la implementación es explicada por la falta de cumplimiento de las Reglas de Operación.

\section{Estudios de caso}

Zacatecas, Jalisco y Michoacán no son solo los estados que más recursos del Programa han concentrado, sino también son los estados que han sido estudiados con mayor frecuencia, quizá porque son estados pioneros en migración y en la implementación del Programa. Algunos estudios coinciden en los efectos positivos del Programa en Zacatecas y Michoacán (Burgess, 2006; González Hernández y González Hernández, 2013; García Zamora, 2007a; Ramírez et al., 2011). Y otros en sus efectos negativos en los estados de Guerrero, Puebla, México e Hidalgo (Díaz Garay, 2009; González Ortiz y Rivera, 2004; Baca Tavira, 2009; Frías et al., 2007).

Por ejemplo Burgess (2006) encuentra que el 
compromiso activo de un club tiende a mejorar la rendición de cuentas del gobierno municipal y de los contratistas, pero que se requiere de la activa participación del comité de obras para, efectivamente, aumentar la gobernanza. Mientras que González Hernández y González Hernández (2013) analizan los efectos del Programa en las capacidades de control social en sus dimensiones de transparencia, sancionabilidad y responsividad, y muestran cambios diferenciados para Zacatecas. La trasparencia ha aumentado, porque Contraloría Interna participa activamente en la fiscalización de los recursos estatales y municipales y es parte de Comité de Evaluación y Atención a Migrantes (COVAM), además de que la información sobre avances y montos de los proyectos es pública (mediante una página web). Mientras que en sancionabilidad y responsividad no hay avances importantes.

García Zamora (2007a) y Ramírez et al. (2011) analizan los proyectos productivos ejecutados bajo el Programa 3x1, el primer autor señala los posibles riesgos de implementar un programa de tipo productivo en un esquema de coinversión social, mientras que, años después, Ramírez et al. (2011) dan cuenta de las formas organizativas que toman los proyectos productivos exitosos en algunas localidades de Zacatecas.

Para Jalisco, el estudio de Valenzuela (2007) da cuenta de los problemas que surgen entre autoridades municipales y migrantes organizados por la definición de las obras, así como la falta de institucionalización en la implementación del Programa, ya que depende de las relaciones personales con las autoridades locales y con los migrantes en los Estados Unidos. La investigación además da cuenta de las negociaciones, fuera de las Reglas de Operación, para obtener el aval del club y poder para realizar alguna obra. Esto incluye la aprobación de proyectos que son de interés del presidente municipal en turno.

En el caso del estado de Guerrero, estado pionero del Programa en su versión 2x1, Díaz Garay (2009) analiza los efectos en las localidades participantes y concluye que es positivo, pero insuficiente en lugares con alta marginación y en extrema pobreza, porque no ha logrado crear inversiones y empleos suficientes para reducir el flujo migratorio ${ }^{5}$,

\footnotetext{
${ }^{5}$ Aspecto que también señala Mérida Rojas (2012). Ambos autores consideran que el Programa no alcanza sus objetivos porque no reduce la migración, pero esto está fuera de los alcances (y objetivos) del Programa, ya que está diseñado bajo una lógica (gubernamental) de aprovechamiento de las remesas y no de reducción del flujo migratorio, podría esperarse en el largo plazo este efecto, siempre y cuando el Programa influyera en el desarrollo local y, en consecuencia, este desarrollo desincentivara la migración. Pero, primero, se tendría que mostrar que el Programa contribuye al desarrollo local. Ruiz-Ochoa (2009) incluso va más lejos al afirmar que
}

además señala que hay malversación de fondos, falta de trasparencia, fracturas entre grupos; que no permiten conjugar los diferentes intereses para realizar algún proyecto, y desconocimiento generalizado del Programa.

Otros estudios, analizan el Estado de México y Puebla, estados donde se reporta poca participación en el Programa. González y Rivera (2004) encuentran un amplio desconocimiento del funcionamiento de dicho programa y poca participación de migrantes organizados. Los autores consideran que esto ocurre porque es una propuesta unilateral, basada en la experiencia zacatecana, que no responde a las necesidades de los migrantes del Estado de México ni de Puebla. Documentan que, en algunos años, el gobierno estatal no realizó las aportaciones correspondientes y en algunos proyectos no hubo participación de los clubes de migrantes.

Vega Briones (2004) y Baca Tavira (2009) coinciden en la falta de información sobre el Programa y en la incipiente participación de los migrantes del Estado de México. Mientras que el estudio de Mérida Rojas (2012) en dos municipios mexiquenses, muestra que en Coatepec Harinas la continuidad del Programa se explica por la iniciativa de las autoridades municipales y la aportación de los familiares de los migrantes. Mientras que en Texcaltitlán hubo mayor participación del club de migrantes, pero la aportación financiera no se hizo si no que fue sustituida por mano de obra aportada por los parientes de los migrantes.

En el Estado Hidalgo, Frías et al. (2007) sostienen que el Programa fue utilizado para disminuir los costos de construcción de las obras de infraestructura de los Ayuntamientos. A partir de un estudio de los municipios del estado que concentran la mayor inversión del Programa, entre 2004 y 2005, los autores muestran que la falta de organización de clubes de migrantes repercute en el reajuste, hecho por los presidentes municipales, en la aportación de los recursos destinados al Programa.

Por ejemplo, en San Jerónimo, Hidalgo, el proyecto fue impuesto por el presidente municipal y la aportación del club de migrantes fue sustituida con recursos de los habitantes de la comunidad, quienes fueron obligados a contribuir económicamente y bajo la amenaza. En otras localidades, los proyectos son ajenos a los intereses de la comunidad, hay desaprobación de las obras, no existen los clubes de migrantes y falta comunicación con las autoridades municipales (Frías et al., 2007). Entre los efectos

no se puede esperar que el Programa reduzca la migración, porque "no puede considerarse una estrategia de desarrollo de alcance estratégico para el progreso y el mejoramiento duradero de la competitividad local" (Ruiz-Ochoa, 2009: 16). 
positivos del Programa, los autores señalan la disminución de costos de construcción de infraestructura básica para las administraciones municipales, pero esto no forma parte de los objetivos del Programa.

De esta manera, los estudios cualitativos documentan diversidad y complejidad en la implementación del Programa 3x1 para Migrantes a nivel municipal, es en este nivel donde se definen los procesos. Como se ha mostrado, en algunos casos los estudios reportan mejores procesos de rendición de cuentas, trasparencia y participación ciudadana, es decir el Programa es considerado como un mecanismo de gobernanza local (Burgess, 2006; González Hernández y González Hernández, 2013). En otros lugares, la implementación del Programa se traduce en malversación de fondos, aumento del control de las autoridades municipales, baja participación de la sociedad civil y agudización de fracturas internas en las localidades y municipios (Baca Tavira, 2009; Díaz Garay, 2009; Frías et al., 2007; Valenzuela, 2007). Sin embargo, estos estudios carecen de un análisis sistemático de las implicaciones de esta heterogeneidad en los resultados del Programa.

\section{Metodología}

Los datos cualitativos que se presentan a continuación son parte de una investigación más amplia sobre clubes de inmigrantes mexicanos realizada en Los Ángeles, California. Para este artículo se utilizaron sólo 13 entrevistas, correspondientes a 13 clubes, de un total de 48 entrevistas semiestructuradas y a profundidad que se hicieron durante el trabajo de campo. Los informantes fueron seleccionados por pertenecer a algún club de inmigrantes y por haber participado en el Programa $3 \times 1$. Se entrevistó a hombres y mujeres, aunque por la composición de los clubes se obtuvieron más entrevistas de varones.

Los clubes analizados pertenecen a su vez a alguna federación, que es una asociación de segundo nivel que los representa y se forma a partir de la identificación con el estado mexicano de procedencia. En este sentido, la investigación sólo indaga en las experiencias de clubes federados, porque hay clubes que no se inscriben en federaciones y participan en el Programa 3x1.

En este caso, se eligió a clubes de las federaciones de Zacatecas, Jalisco y Chiapas, porque son lugares con antecedentes migratorios y procesos asociativos diferentes que permiten la comparación. Zacatecas y Jalisco son estados con antiguos antecedentes migratorios, al menos desde inicios del siglo XX, su migración es más familiar y permanente, mientras que Chiapas tienen una migración reciente, de finales del siglo XX, en su mayoría indocumentada y masculina.

Además se entrevistaron a tres funcionarios del Instituto de los Mexicanos en el Exterior (IME) relacionados con la población inmigrante a través de diferentes programas, y a la representante de la Secretaría de Desarrollo Social en Los Ángeles.

La información de campo se obtuvo entre 2010 y 2012 y se clasificó para su análisis en categorías siguiendo los procesos de codificación abierta, axial y selectiva, es decir se utilizó la teoría fundamentada, propuesta por Strauss y Corbin (2002), sólo en su dimensión procedimental, para formar esquemas interpretativos a partir de las diferentes experiencias de los informantes.

Por otro lado, se analizaron las Reglas de Operación del Programa desde 2002 y hasta la actualidad (2018) para identificar los cambios en las regulaciones y en las competencias de cada actor, de esta manera las Reglas de Operación fueron el referente formal para comparar de las prácticas concretas de los actores locales.

\section{Población atendida, población objetivo y el lugar de nacimien- to}

El Programa ha tenido varias modificaciones desde 2002, en el nombre, los objetivos, la cobertura y la población objetivo. Por un lado, los cambios en el objetivo general reflejan los diferentes alcances que se le han adjudicado y estos, a grandes rasgos, son: "mejorar la calidad de vida" (2002-2003 y 2009-2012), promover la "equidad y enfoque de género" (2004-2007), promover el "desarrollo social" (2008) y "fortalecer la participación social para impulsar el desarrollo comunitario" (2015-2018) (González Rodríguez, 2011; SEDESOL, 2003, 2004, 2005, 2011, 2013a, 2013b, 2014, 2015, 2016, 2017).

En cuanto a los objetivos particulares, estos se mantuvieron sin cambios hasta 2008, sin embargo hay uno que fue señalado, como hemos mostrado anteriormente en las evaluaciones, como un punto crítico y es el que refería a: "promover que las propuestas de inversión se canalicen a las comunidades de alta migración y pobreza" (Diario Oficial de la Federación, 2007: 3). En 2009 solo se acotó a las "comunidades de origen" de los migrantes, posteriormente, a "promover" o "generar" desarrollo comunitario en las "localidades seleccionadas por 
los migrantes" (2014-2018).

Con respecto a la cobertura se amplió territorialmente de 31 estados a las 32 entidades federativas y de localidades en "zonas marginadas" a "localidades seleccionadas por los clubes u organizaciones de migrantes" (2014-2018). La población objetivo ha tenido también varios ajustes, estos han sido en la unidad de atención: de 2002 a 2012 la población objetivo eran las personas y a partir de 2013 son las localidades.

También se modificaron los criterios que acotan a la población objetivo: personas que habitan en localidades "en condiciones de pobreza extrema" (2002), "en condiciones de pobreza" (2003-2007), "seleccionadas por los migrantes" (2008), "en condiciones de pobreza, rezago o marginación” (2009), "que presentan condiciones de rezago en materia de infraestructura, servicios y necesidades vinculadas con la actividad económica" (2010-2012), localidades "seleccionadas para invertir" (2013), "seleccionadas por los clubes u organizaciones de migrantes" (2014-2017) y, en 2018, se incorporó nuevamente el criterio de marginación (grado muy alto, alto o medio) e intensidad migratoria (muy alto, alto o medio).

Muchos de los ajustes responden a las recomendaciones de las evaluaciones, así como a las presiones de grupos de migrantes (que participan activamente) y de otros actores políticos ${ }^{6}$. Sin embargo, no se ha logrado salvar la contradicción entre los criterios de la población objetivo (pobreza y marginación) y las características de las localidades atendidas, porque, por un lado, el Programa es subsidiado con los recursos del Ramo Administrativo 20, correspondientes a Desarrollo Social.

Al estar ubicado en la cartera de programas de la SEDESOL tiene como propósito contribuir a la reducción de la pobreza y la marginación, como está estipulado en los recursos del Ramo 20: "Las políticas y estrategias de desarrollo social están enfocadas a la disminución de los índices de pobreza y marginación a través del desarrollo de capacidades que fomente la inclusión social" (Secretaría de Hacienda y Crédito Público, 2017: 2). De ahí que esta prioridad aparezca consistentemente en diferentes secciones de las Reglas de Operación del Programa, a pesar de las modificaciones realizadas en los objetivos particulares y en la población objetivo, finalmente es un criterio del Ramo 20 que no puede ser omitido.

\footnotetext{
${ }^{6}$ Congresos estatales y legisladores de diferentes fracciones partidistas han presentado propuestas en el Congreso de la Unión para: la asignación de más recursos, la promoción del Programa, la promoción de las organizaciones de migrantes, la modificación de las ROP o para solicitar que las Reglas no se modifiquen (Ver González Rodríguez, 2011).
}

Por otro lado, la forma de gestión y la participación de los migrantes, antes de que se convirtiera en un programa federal, responden a problemas diferentes a los estipulados en el Ramo 20. Como ya han documentado otros trabajos, la gestión e inversión de recursos de los migrantes en las localidades de origen comienza en la década de los sesenta, en las comunidades de migrantes con una mayor tradición migratoria como Zacatecas (Moctezuma y Pérez Veyna, 2006). Además, es una forma frecuente de relación con la comunidad de origen que también se encuentra en otras comunidades de migrantes mexicanos, con menos antigüedad en la migración a los Estados Unidos y no necesariamente bajo el Programa 3x1 para Migrantes (Frías et al., 2007; Imaz, 2004).

Los migrantes mexicanos, en los Estados Unidos, comenzaron a realizar eventos sociales para recaudar fondos y con estos recursos financiaron obras de infraestructura, ayudaron a personas en problemas y/o patrocinaron fiestas en la localidad de origen. Dado que los recursos eran aportados totalmente por los migrantes, organizados en clubes o no, ellos tomaban la mayor parte de las decisiones. Las obras de infraestructura se hacían con total independencia de las autoridades municipales y no se negociaba ni el tipo de obra ni la localidad en donde se iba a ejecutar el proyecto. Sin embargo, en muchas ocasiones, la obra era propuesta por los habitantes de las localidades de origen, porque los migrantes recibían (y reciben) solicitudes de éstos, en este sentido, no elegían (ni eligen) totalmente el tipo de proyecto a financiar.

El incentivo de los migrantes para aportar recursos, recaudar fondos e invertir en las localidades de México proviene de los lazos familiares y de las vivencias compartidas en un territorio, de manera que la solidaridad se genera por la pertenencia a un mismo lugar. Los problemas de coordinación y participación de toda acción común (o colectiva), en el caso de los migrantes, se resuelven a partir del lugar de nacimiento, es decir de la localidad en un sentido amplio, con su historia y vivencias compartidas referenciadas territorialmente. Por ello, la organización de migrantes, en clubes o en otro tipo de asociación, es por localidad de origen. 
En una ocasión esta persona (...) nos llamó a unas cuantas personas y sin decirnos para qué, nos dice un día: oigan, que les parece si, ¿no les gustaría que hiciéramos algo por Santa Rosa? Porque todo mundo lo conocemos por Santa Rosa, y pues básicamente todos así, al instante, todos dijimos: ¡Pues, claro! Porque de hecho todos desde que salimos de allí, de nuestro Santa Rosa, pues, nos venimos con una idea de tratar de mejorarlo. No venimos con las ideas claras de qué manera hacerlo, pero todos venimos con esa intención.

(Entrevista realizada a persona del Club Santa Rosa y Santa Gertrudis el 18 de octubre de

(...) mucho de esto de ayudar es porque crecimos allá, porque queremos el pueblo, y porque, a mí, me gusta. Porque llegas y ves qué bonita está la escuela, qué orgullo, al menos a mí, a mí me da muchísimo orgullo saber que muchas de las escuelas de Jalpa es gracias al trabajo que muchos de nosotros hemos hecho aquí como familia.

(Entrevista realizada a persona del Club Familias Unidas por Jalpa el 11 de julio de 2012.)

El interés compartido en mejorar, contribuir, retribuir o ayudar a las personas de la localidad de origen es el elemento que hace posible la organización de los migrantes en los Estados Unidos en clubes. Entonces, el lugar de nacimiento es el que posibilita la conjunción de esfuerzos y recursos individuales en un proyecto común, y es el lugar donde los migrantes están dispuestos a invertir sus recursos.

(...) siempre tiene uno en mente de ayudar a su gente que queda atrás en México, en los pueblos chicos hay muchas necesidades y en los pueblos rurales más, porque están retirados de un pueblo grande, donde recibe más entrenamiento, puede trabajar la gente por un sueldo, y en esos pueblitos chiquitos, ranchos, no, no hay sueldos. Se trabaja por ayudar a alguien y le ayudan a la vez. $\mathrm{Y}$, entonces, nosotros teníamos ganas de ayudar a nuestra gente, sabíamos que podíamos y nos juntamos un grupo.

(Entrevista realizada a persona del Club Fátima de Majadas el 29 de agosto de 2012.)
Y yo pensé también, cuando me vine, pensé que un día regresaría a ayudar a nuestra gente si yo tenía éxito en mi vida. Entonces, cuando me invitó [a formar el club], pues no lo dudé, yo tenía ya cuatro o cinco años con mi negocio propio y bueno, pues, no nos fue mal, y pensé que era manera de poder pagarle algo a la comunidad, regresar algo a la comunidad que nos vio nacer.

(Entrevista realizada a persona del Club Santa Juana el 21 de septiembre de 2012.)

En este sentido, los esfuerzos de los migrantes están articulados por el lugar de nacimiento y es el incentivo para invertir sus recursos en México, de manera que, cuando se diseñan las Reglas de Operación del Programa, bajo los criterios de la SEDESOL, se genera una contradicción de fondo entre las condiciones de pobreza y marginación; prioridad de atención de la Secretaría, y las características de las localidades de origen de los migrantes; incentivo de la participación de las personas.

(...) si yo soy de Juchipila, ¿por qué voy a hacer una obra en Mazapil? No, no, tiene sentido. No es que no queremos ayudar a los municipios más rezagados, no hay problema, pero, entonces, díganos, a nosotros, que ya no vengamos y háblenles a los de Mazapil para que vengan. ¿A qué venimos nosotros si no nos van a dar las obras? Si no nos van a apoyar. Es que ustedes ya no necesitan, dicen, es que ustedes están muy adelantados, ya tienen servicios de agua, ya tienen luz, ya tienen todo, ustedes ya no, vamos a darle a los que no tienen. Yo estoy de acuerdo, si es el pensamiento del gobierno del estado, de acuerdo, pero (...) mejor no venimos y que le hablen a los estados o a otros municipios que no se presentan o que no se organizan.

(Entrevista realizada a persona del Club Remolino el 8 de julio de 2012.)

Desde la visión de los actores gubernamentales no se entiende por qué los migrantes no están dispuestos a gestionar recursos para las localidades más marginadas o por qué los migrantes de estas localidades no participan en el Programa. Desde la perspectiva de los migrantes, la localidad de nacimiento es el interés central de la aportación voluntaria de recursos y tiempo. Además, como han reportado otras investigaciones, la migración de mexicanos a los Estados Unidos no ocurre en aquellas localidades más pobres del país, por ello, los altos índices de migración internacional no están acompañados de altos índices de marginación y/o pobreza, sino que las localidades expulsoras tienen un grado medio de marginación (Raccanello y López Velázquez, 2008; CONAPO, 2010). 
Cabe señalar que la organización de migrantes es altamente costosa en términos sociales y económicos, la recaudación y gestión de recursos en los Estados Unidos requiere de un tipo de migrante que cuenta con un excedente y está dispuesto a invertirlo en México. Estas características no las tienen los migrantes recién llegados, cuya preocupación es resolver las necesidades básicas de vida como el empleo y vivienda. Si bien se trata de una acción solidaria y hasta altruista, porque no tiene una retribución inmediata en los mismos términos, es facilitada por migrantes establecidos (con independencia del estatus migratorio), que en su mayoría son procedentes de regiones con migración antigua (PérezSoria, 2015).

De esta forma, la tensión entre población objetivo y población atendida provienen de: a) las regulaciones del Ramo 20, donde se inscribe el Programa 3x1 para Migrantes, b) el incentivo de los migrantes para gestionar e invertir sus recursos: el lugar de nacimiento es el que originó la organización de migrantes y posibilitó la gestión de recursos, con independencia de las autoridades gubernamentales, y es el que promueve la participación en el Programa, y c) las características de las localidades expulsoras de población (marginación media) y de los migrantes, sobre todo en los Estados Unidos (con recursos suficientes para invertir en proyectos en México). Es importante entender que no migran los más pobres y tampoco pueden enviar remesas colectivas los migrantes más pobres; preocupados y ocupados por la sobrevivencia en el lugar de residencia y por los familiares del lugar de origen.

\section{Centralidad de otros actores: presidentes municipales y po- blación local}

Los criterios para participar en el Programa, establecidos en las Reglas de Operación, son: que los migrantes estén organizados y cuente con Toma de Nota vigente, que el club u organización de migrantes "decida apoyar un proyecto", "designe a un representante en México" y "demuestre solvencia económica para la aportación al proyecto que propone", en el caso de los de proyectos de infraestructura social, servicios comunitarios o educativos (SEDESOL, 2017).

Los migrantes son los promotores y patrocinadores (del 25 por ciento) del proyecto, la población de la localidad, además de ser la población beneficiada, funge como contraloría social, porque de ahí surge el Club Espejo, que se encarga de vigilar la correcta ejecución del proyecto, y el gobierno municipal se encarga de ejecutar la obra y de patrocinarla (con otro 25 por ciento) (Ahumada Lobo y Asociados, 2016; SEDESOL, 2017). En la implementación del Programa, la relación entre estos tres actores (migrantes, población local y autoridades municipales) define el proceso y los resultados.

\section{Migrantes organizados y presidentes municipales}

La relación entre el club de migrantes y las autoridades municipal puede ser de cooperación, de manera que participan como socios en el logro de proyectos, o de conflicto por la selección de la obra ${ }^{7}$, la aprobación, la ejecución y la adjudicación de la misma.

Si de repente el presidente del municipio no considera que es buena idea apoyar el proyecto que va por el club, ya ahí nos topamos con el primer problema, pero mientras el presidente dice: está bien lo aprobamos, yo te apoyo; porque el presidente del municipio lleva un 25 por ciento en apoyo también, entonces, si él considera que es bueno, lo apoya, por ahí no hay problema.

(Entrevista realizada a persona del Club Santa Rosa y Santa Gertrudis el 18 de octubre de

La relación se complica con el cambio, cada tres años, de los presidentes municipales, porque, en un mismo municipio, la continuidad en el Programa es incierta, depende del tipo de relación que se establezca entre el grupo de migrantes y el presidente muncicipal:

${ }^{7} \mathrm{El}$ estudio de Valenzuela (2007) muestra la opinión negativa de algunos presidentes municipales sobre los criterios de los migrantes para elegir la obra. Esto también está reportado en la primera evaluación del Programa Iniciativa Ciudadana, donde se menciona que el presidente de Juchipila consideraba que "sería mucho mejor si los migrantes no intervinieran en la decisión de las obras" (SEDESOL, 2003: 186-187). 
Mira, te voy a decir que, el actual presidente que acaba de salir, excelente relación, creo es el mejor presidente, muy buena relación. Apoyando todos los proyectos y actividades que nosotros queremos realizar en nuestro municipio. Las otras administraciones pasadas, este, cuando iniciamos como club, estuvimos un año con el otro presidente, excelente, después tuvimos otra administración de otras personas, yo te podría decir que ahí si tuvimos un poquito de problemas, porque al club no nos veían como socio, si no que nos veían como un rival.

(Entrevista realizada a persona del Club Cañadas de Obregón el 19 de octubre de 2012.)

El problema es aún más agudo cuando los proyectos son grandes y se dividen en etapas, porque inician en un periodo, con un presidente municipal, y para el siguiente año, el cambio en la presidencia del Ayuntamiento, dificulta la continuidad de la obras. Además, las campañas políticas, a nivel municipal, crean divisiones en las organizaciones de migrantes, porque los candidatos buscan capitalizar el apoyo de estos grupos y las personas que los conforman tienen preferencias partidistas distintas ${ }^{8}$.

(...) el problema es cuando vienen las elecciones de cada tres años de los presidentes municipales, ahí es donde empiezan los problemas, ¿por qué? Porque si ven que un grupo está bien y está trabajando bien, sin partido de ningún tipo, lo hacen de un partido, del que sea, del que se meta primero, del que los logra convencer: miren, vénganse para acá y acá vamos a estar fuertes. Y muchas veces los separan, no toda la gente jala, pero sí los logran separar.

(Entrevista realizada a persona del Club Remolino el 8 de julio de 2012.)

Sea que la relación entre actores se caracterice por la cooperación o por el conflicto, la incidencia de los gobiernos municipales en el Programa, sin participación activa de los migrantes organizados, se traduce en un problema de centralización en la toma de decisiones y falta de trasparencia en el uso de los recursos públicos.

El gobierno municipal es la instancia local directamente involucrada en el ejecución del proyecto, esto implica el manejo de los recursos financiero, la elección del contratista que ejecutará la obra y la

\footnotetext{
${ }^{8}$ Recuérdese que se organizan a partir del lugar de nacimiento, no por preferencias políticas en México o en los Estados Unidos. A pesar de que el Programa se federalizó en 2002, cuando la presidencia de la República estaba ocupada por un candidato del Partido Acción Nacional (PAN), esto no implica que los migrantes que participan en el Programa, sean simpatizantes de este partido político.
}

compra de materiales, por tanto, hay casos donde el presupuesto de la obra es deliberadamente elevado, por encima de los costos reales, con el propósito de recuperar la inversión que hace el Ayuntamiento o para sustituir la aportación del club de migrantes. Esto ocurre cuando hay baja o nula participación de migrantes y de población local.

Al grupo de migrantes que hace la solicitud, firma los documentos y no aporta el 25 por ciento de la obra se le conoce como "club aval", y puede ocurrir que un grupo de migrantes que participa activamente, en ocasiones, funja como club aval en algunos proyectos. También hay otros grupos que solo son avales de los proyectos del gobierno municipal, es decir, que nunca han seleccionado la obra ni aportado los recursos.

Ýa nada más nos llevan a certificar. Lo que escuchabas [en la reunión sobre] las Reglas [de Operación] era mucho en ese sentido, de que muchas veces yo como club verdadero propongo, pero tú como presidente municipal te pusiste de acuerdo con fulano, y fulano ni club es, pero se sentó a tomar café con alguien y se inscribió como club. Y nada más te dan la aplicación y tú consigues el dinero con el contratista, lo consigues tú mismo, o haces lo que quieras para bajar dinero.

(Entrevista realizada a persona del Club La Villita el 17 de agosto de 2012.)

A veces está practica es el resultado de una negociación entre migrantes y presidente municipal, es decir se llega al acuerdo de "avalar" una obra de interés del Ayuntamiento a cambio de aprobar un proyecto de interés de los migrantes, como ha documentado Valenzuela (2007). En otras situaciones es el presidente municipal el que busca directamente a un grupo de migrantes o a una persona para gestionar los recursos del Programa.

(...) cuando vino un presidente municipal que en aquel tiempo, me dijo: necesito a alguien que le interese un pozo profundo, una presa. No, pues, un señor andaba queriendo hacer uno. No, pues, háblale, que venga. Y le hable, vino, y lo invité a que hablara con el presidente.

(Entrevista realizada a persona del Club Remolino el 8 de julio de 2012.)

En la opinión de los entrevistados el problema se ha agudizado, porque al inicio del Programa la mayor parte de los grupos de migrantes y de los presidentes municipales aportaban sus respectivas cuotas para la realizar las obras. Posteriormente, 
por un lado, el interés de los presidentes municipales en conseguir recursos extras, para sus administraciones, encontró en el Programa una fuente de ingresos estatales y federales.

El 3x1, el 2x1 estuvo bien, había dinero suficiente, el 3x1 empezó bien, pero ya ahorita a estas alturas no es suficiente y no es suficiente porque al principio todos los proyectos eran reales, los presidentes municipales no daban importancia al 2x1 o $3 \times 1$, es un proyecto, está bien que nos manden dinero y lo aplican, pero con el paso del tiempo dijeron: ¡Ah, cabrón!, aquí hay negocio. Entonces, los últimos presidentes siempre han buscado negocio en el 3x1, ya no te dejan trabajar como solían hacerlo.

Por otro lado, los migrantes han disminuido su participación en el Programa, acorde a las Reglas de Operación, por la dificultad creciente de recaudar fondos en los Estados Unidos. Estos fondos, muchas veces, no alcanzan para cubrir la aportación del club y negocian con los presidentes municipales o con la población local.

Ya no puedes hacer eventos, porque no queda dinero, entonces, debido a eso, ellos [los presidentes municipales] ya agarraron fuerza, ¿verdad? La parte del club la pone el presidente municipal, la parte del presidente municipal también viene de allá, y todo viene de allá (...). Entonces, cómo llego con el presidente municipal: no, esto no, esto sí. Pues no, ¿Tú qué pusiste? No pues nada más la firma. Está bien difícil, y antes no, antes sí mandaba uno el dinero real de aquí, ¿verdad?, ahorita ya con la economía que se puso aquí tan mal, pues ya los clubes no pueden hacer fondos.

(Entrevista realizada a persona del Club Social Atolinga el 21 de agosto de 2002.)

Esto ha hecho que, en el los dieciséis años que lleva operando el Programa, los presidentes municipales tengan mayor injerencia en la toma de decisiones en torno a los recursos del Programa, sin rendición de cuentas, porque los otros actores, que están contemplados en el diseño (migrantes organizados, comité de obras o club espejo, instancias estatales y federales), han disminuido su intervención o carecen de mecanismos efectivos de control y vigilancia.

\section{Migrantes organizados y población de las localidades beneficiadas}

La relación entre migrantes organizados y población local tiene otras características. La población de las localidades beneficiadas también suele definir la obra, pero esto no genera conflicto, como ocurre con los presidentes municipales, porque, como ya hemos señalado, en muchas ocasiones, son las personas o los grupos de la localidad de origen los que hacen la solicitud al club de migrantes, para que se realice algún proyecto dentro del Programa 3x1.

Ya les ayudamos también un año con computadoras, también ya tienen computadoras, y ella dice: mira, [la escuela] está toda descarapelada, los baños están mal, ocupamos otra [obra] de $400 \mathrm{mil}$ pesos, para que nos ayuden.

(Entrevista realizada a persona del Club Remolino el 8 de julio de 2012.)

En otras ocasiones, los migrantes eligen la obra, sin consultar a los pobladores de la localidad, como está estipulado en las Reglas de Operación, es decir estas Reglas no contempla la posibilidad de que la obra sea definida por las personas de la localidad ni que participen en el proceso de selección del proyecto. Sin embargo, cuanto esto ocurre genera desconfianza y poca involucramiento de la población.

\begin{abstract}
¿Y la gente de allá cómo responde?
Es una buena pregunta, la mayoría de las personas, se esperaría que la respuesta fuera: ¡Oh!, que todo mundo lo tomara de una manera bien, como buena idea, bien agradable, pero la verdad es que no es así, siempre esto, como cualquier otra organización, grupo organizado, siempre se encuentra con las personas que tratan de ponerte trabas o simplemente no cooperan, no se unen, no ayudan, no se unen, entonces, no fue la excepción con nosotros.
\end{abstract}

(Entrevista realizada a persona del Club Santa Rosa y Santa Gertrudis el 18 de octubre de 2012.)

Ahora, hay casos donde la población local elige la obra, busca al club para que avale el proyecto y aporta el 25 por ciento (o menos). Esto cambia el papel de la población: de beneficiada a patrocinadora del proyecto. Es decir, hay personas interesadas en que se realice algúna obra, aportan el monto correspondiente al club, solicitan, al igual que hacen los presidentes municipales, el aval de algún club e inician el proceso de gestión dentro del Programa. 
Y en realidad no es tanto el dinero, o sea, nosotros es muy poco lo que aportamos. Casi la mayoría de las veces las escuelas se juntan por nosotros y ellos aportan casi todo el dinero, pero nos usan a nosotros para hacer la [obra], para tener acceso al Programa $3 \times 1$. Muchas veces [ocurre esto] y sobre todo ahorita que económicamente hay crisis. Antes, nos dábamos nosotros el lujo de hacer un baile con 500 personas, ahorita haces un baile y van 60,80 personas.

(Entrevista realizada a persona del Club Familias Unidas por Jalpa el 11 de julio de 2012.)

De manera que el uso del "club aval" también se encuentra en la población de la localidad de origen y ocurre fuera de las Reglas de Operación, pero la diferencia radica en las consecuencias que genera: cuando la población local elige la obra y aporta recursos económicos, participa activamente en el seguimiento de la misma, tiene control de los gastos y de los materiales empleados, es decir se involucra en todo el proceso de ejecución.

Es cuando el Comité de Obras o Club Espejo, formado por la población local, realiza actividades de contraloría social efectiva, porque tiene interés en el proyecto y tiene recursos invertidos en la obra. Por tanto, aunque los dos escenarios ocurren fuera de las ROP, en este segundo caso hay mayor rendición de cuentas, por el papel activo de la población local. En este sentido, coincidimos con Burgess (2006) cuando afirma que la participación activa de la población local en el Programa es fundamental para la gobernaza. El interés de la población y la presencia física en el lugar donde se ejecuta la obra, aumenta la vigilancia sobre los recursos y sobre las decisiones de la autoridades municipales.

\section{Reflexiones finales}

En este trabajo se ha mostrado cómo el análisis a nivel micro contribuye a explicar algunas situaciones reportadas por los estudios globales del Programa 3x1 para Migrantes. La ejecución de los proyectos se caracteriza por la negociación, la cooperación, el conflicto y la imposición de intereses entre actores: migrantes organizados, población local y autoridades municipales. Las relaciones entre estos actores definen el proceso de implementación del Programa, muchas situaciones y acuerdos ocurren fuera de las Reglas de Operación y sus resultados son divergentes.

A pesar de que las ROP señalan que son los migrantes organizados los actores centrales de los proyectos, cuando esto no ocurre y otro actor toma el papel protagónico en el Programa se producen efectos diferentes y antagónicos: mayor concentración en la toma de decisiones cuando son las autoridades municipales las que desplazan el papel de los migrantes, y mayor rendición de cuentas cuando es la población local.

El club aval se ha convertido en una práctica recurrente en el Programa, no solo en aquellos estados de la República que tienen poca participación y una migración reciente, sino incluso en aquellos estados pioneros del Programa como Zacatecas y Jalisco. Esto ha ocurrido, entre otros factores, por la dificultad creciente de recaudar recursos en los Estados Unidos.

Ante esta situación, otro actor realiza la aportación correspondiente al grupo de migrantes y, con ello, la relación entre los actores, y la toma de decisiones, se redefine. Cuando es la población local la que aporta los recursos, se transita de población beneficiada a actor patrocinador del proyecto, cuando es el gobierno municipal el que cubre el aporte de los migrantes, en el mejor de los escenarios, incrementa su aporte al 50 por ciento, porque en otros casos eleva el presupuesto del proyecto para cubrir ficticiamente el aporte de los migrantes.

Cabe señalar que las aportaciones ficticias y las altas cotizaciones no son prácticas que se encuentren solo en el Programa 3x1 para Migrantes, también se han reportado en la implementación de otros programas gubernamentales en México. La centralidad de los presidentes municipales en el Programa produce el escenario más crítico, no sólo porque ocurre fuera de las Reglas de Operación, sino porque, como mostramos, tiene efectos negativos en la rendición de cuentas.

Ahora, por más que se ajusten las Reglas de Operación, las localidades atendidas corresponderán a las localidades de origen de los migrantes que deciden participar en el Programa, independientemente de los índices de pobreza y de marginación, porque no hay otro incentivo para los migrante, que envían remesas colectivas, que mejorar el lugar de nacimiento. De manera que, el desfase entre población atendida y población objetivo del Programa, es producto de una incompatibilidad entre los objetivos de la SEDESOL y los intereses (y objetivos) de los migrantes organizados.

La forma de gestión que los grupos de migrantes implementaron por más de cuatro décadas, y que dio origen al Programa 3x1 para Migrantes, respondía a un conjunto de problemas y necesidades identificados por los migrantes en sus localidades de origen, cuando esta propuesta salta a la agenda gubernamental, en 2002, se incorpora como alter- 
nativa, es decir como el mecanismo de solución a un problema definido por la SEDESOL: contribuir a reducir la pobreza y la marginación.

En este sentido, la solución, propuesta por la sociedad civil migrante, se conjuntó con el problema identificado por la SEDESOL, pero los grupos de migrantes ya tenían identificado previamente un problema y no es el mismo que define la Secretaría de Desarrollo Social. De esta forma, las dos lógicas subyacen en el diseño y en la implementación del Programa, en momentos resultan incompatibles y aumentan los problemas de cooperación entre actores.

Finalmente, este trabajo busca establecer un puente entre comunidades académicas, porque la literatura sobre el Programa 3x1 para Migrantes es abundante, pero parcial. Mientras que los estudios a nivel micro, de localidades y municipios, prescinden de incorporar el análisis de las Reglas de Operación, como un marco normativo institucional que acota las posibilidades del Programa, orienta las relaciones y establece obligaciones entre actores. Las evaluaciones omiten la revisión de los estudios de caso que mucho aportan sobre los problemas, conflictos y retos del Programa.

\section{Referencias}

Ahumada Lobo y Asociados (2016). Evaluación Complementaria de Resultados del Programa 3X1 Para Migrantes 2013-2014.

Baca Tavira, N. (2009). Migración y gobierno: Atención a migrantes internacionales en el estado de méxico. Gaceta Laboral, 15(3):05-35.

Burgess, K. (2006). El impacto del 3x1 en la gobernanza local. En Fernández de Castro, R., García Zamora, R., y Vila Freyer, A., editores, El programa $3 x 1$ para migrantes: ¿Primera política transnacional en México?, pp. 99-118. Miguel Ángel Porrúa; Universidad Autónoma de Zacatecas; Instituto Tecnológico Autónomo de México, México D.F.

CIDE (2008). Evaluación externa del Programa 3x1 para Migrantes 2007. Secretaría de Desarrollo Social.

CONAPO (2010). Índices de intensidad migratoria México-Estados Unidos.

CONEVAL (2008). Evaluación Específica de Desempeño 2008.

CONEVAL (2010). Evaluación Específica de Desempeño. Datos generales del programa.
CONEVAL (2011). Evaluación Específica de Desempeño del Programa 3 x 1 para Migrantes 2010-2011.

CONEVAL (2015). Informe de la evaluación específica de desempeño 2014-2015. Valoración de la información de desempeño presentada por el Programa. Programa 3x1 para Migrantes.

Délano, A. (2006). De la no intervención a la institucionalización: la evolución de las relaciones Estado-diáspora en el caso mexicano. En González Gutiérrez, C., editor, Relaciones Estado-diáspora: la perspectiva de América Latina y el Caribe. Tomo II, volumen 2, pp. 145-183. Miguel Ángel Porrúa, UAZ, Secretaría de Relaciones Exteriores, Instituto de los Mexicanos en el Exterior, ANUIES, México D.F.

Diario Oficial de la Federación (2007). Acuerdo por el que se emiten y publican las Reglas de Operación del Programa de Atención a los Adultos Mayores de 70 anos y más en zonas rurales para el ejercicio fiscal 2007, Tomo DCXLI, No. 19, Sexta Sesión, 28 de febrero de 2007.

Díaz Garay, A. (2009). Transnacionalidad social mexicana. alcances y limitaciones del programa $3 \mathrm{x} 1$ para migrantes en guerrero. Trayectorias, 11(29):117-131.

Faret, L. (2004). Implicarse aquí, con la mirada hacia allá: la organización comunitaria de los guanajuatenses en los Estados Unidos. En Lanly, G. y Valenzuela, B., editores, Clubes de Migrantes Oriundos Mexicanos en los Estados Unidos. La politica transnacional de la nueva sociedad civil migrante, pp. 225-252. Universidad de Guadalajara, Zapopan.

Frías, N., Ibarra, M., y Rivera, L. (2007). La organización comunitaria. Actor ausente en la reglamentación del Programa 3x1 para migrantes en Hidalgo. En Fernández de Castro, R., García Zamora, R., y Vila Freyer, A., editores, El Programa 3x1 para migrantes: ¿Primera política trasnacional en México?, pp. 171-196. LX Legislatura, ITAM, UAZ, Miguel Ángel Porrúa, México D.F.

García Zamora, R. (2007a). El Programa 3 x 1 y los retos de los Proyectos Productivos en Zacatecas. En Fernández de Castro, R., García Zamora, R., y Vila Freyer, A., editores, El Programa $3 x 1$ para Migrantes: ¿Primera política transnacional en México?, volumen 3, pp. 157-170. LX Legislatura, ITAM, UAZ, Miguel Ángel Porrúa, México, D.F.

García Zamora, R. (2007b). El Programa Tres por Uno de remesas colectivas en México: Lecciones y desafíos. Migraciones internacionales, 4(1):165172 . 
García Zamora, R. y Padilla, J. M. (2012). Las organizaciones de migrantes mexicanos en USA y su estrategia de desarrollo local con enfoque transnacional: avances y desafíos. Barataria. Revista Castellano-Manchega de Ciencias Sociales, (13):47-66.

González Hernández, J. R. y González Hernández, G. M. (2013). Rol del agente y estructuras de rendición de cuentas en programas sociales mexicanos. El caso del programa $3 \times 1$ en Zacatecas. Revista chilena de derecho y ciencia política, 4(2):73-101.

González Ortiz, F. y Rivera, L. (2004). Migrantes y políticas públicas. Apuntes desde la experiencia del programa Iniciativa Ciudadana Tres por Uno en los estados de México y Puebla.

González Rodríguez, J. (2011). El programa 3x1 para migrantes. Datos y referencias para una revisión complementaria. Documento de Trabajo Núm. 111. Centro de Estudios Sociales y de Opinión Pública. LXI Legislatura, México, D.F.

Imaz, C. (2004). Poder Político de las organizaciones transnacionales de migrantes mexicanos en sus comunidades. Estudio comparativo de clubes sociales de migrantes en Nayarit-California y Puebla-Nueva York. En Lanly, G. y Valenzuela, B., editores, Clubes de migrantes oriundos mexicanos en los Estado Unidos. La política transnacional de la nueva sociedad civil migrante, pp. 375-422. Universidad de Guadalajara, Zapopan.

Lanly, G. y Hamann, V. (2004). Solidaridades transfronterizas y la emergencia de una sociedad civil transnacional: la participación de dos clubes de migrantes en el desarrollo local del occidente de México. En Lanly, G. y Valenzuela, B., editores, Clubes de migrantes oriundos mexicanos en los Estado Unidos. La política transnacional de la nueva sociedad civil migrante, pp. 127-174. Universidad de Guadalajara, Zapopan.

Leal Jiménez, C. (2008). Gobernanza, clubes de migrantes y Programa 3 x 1: la transparencia y la rendición de cuentas en el manejo de remesas colectivas. Tesis de máster, El Colegio de la Frontera Norte.

Mérida Rojas, A. d. C. (2012). El programa $3 x 1$ para migrantes: estudio de caso de los municipios de Coatepec Harinas y Texcaltitlán. Editorial Académica Española, México.

Meseguer, C. y Aparicio, F. J. (2012). Migration and distributive politics: the political economy of Mexico's 3x1 Program. Latin American Politics and Society, 54(4):147-178.
Moctezuma, M. y Pérez Veyna, O. (2006). Remesas colectivas, Estado y Formas Organizativas de los mexicanos en EUA. En Fernández de Castro, R., García Zamora, R., y Vila Freyer, A., editores, El Programa $3 x 1$ para Migrantes: ¿Primera politica transnacional en México?, pp. 119-138. LX Legislatura, Miguel Ángel Porrúa, ITAM, UAZ., México D.F.

Pérez-Soria, J. (2015). Organización interna de los clubes de oriundos: un análisis desde el lugar de destino. Espiral. Estudios sobre Estado y Sociedad (Guadalajara), 22(64):149-188.

Raccanello, K. y López Velázquez, D. (2008). El programa Tres por Uno para migrantes: un análisis de las localidades receptoras. Escenarios de Población, pp. 113-135.

Ramírez, R., Pérez, O., y Hernández, F. (2011). Los proyectos productivos financiados con remesas y el 3x1 en los municipios zacatecanos y sus formas de organización. Conciencia Tecnológica, (41):13-21.

Red de Consultores Nacionales (2004). Evaluación del Programa Iniciativa Ciudadana 3x1, 2003.

Rodríguez Ramírez, H. (2012). El papel de los migrantes mexicanos en la construcción de una agenda de políticas públicas: El caso del Programa 3x1. Región y sociedad, 24(53):231-257.

Ruiz-Ochoa, W. (2009). Valoración de la estrategia de remesas colectivas para combatir la marginación en México. Ra Ximhai. Revista de Sociedad y Desarrollo Sustentable, 5(1):1-17.

Sánchez, T. y Jiménez, E. (2011). Organización pionera en la construcción y el desarrollo de políticas públicas transnacionales. El caso del Programa $3 \times 1$ para Migrantes.

Secretaría de Hacienda y Crédito Público (2017). Proyectos de presupuesto de egresos de la federación 2017. Estrategia Programática.

SEDESOL (2003). Informe final de la evaluación externa del programa Iniciativa Ciudadana $3 \times 1$, 2002.

SEDESOL (2004). Acuerdo por el que se modifican las Reglas de Operación del Programa Iniciativa Ciudadana $3 \times 1$.

SEDESOL (2005). Acuerdo por el que se emiten y Publican las reglas de Operación del Programa $3 x 1$ para Migrantes.

SEDESOL (2011). Acuerdo por el que se emiten las Reglas de Operación del Programa 3x1 para Migrantes, para el ejercicio fiscal 2012. 
SEDESOL (2013a). Acuerdo por el que se emiten las Reglas de Operación del Programa 3x1 para Migrantes, para el ejercicio fiscal 2013.

SEDESOL (2013b). Acuerdo por el que se emiten las Reglas de Operación del Programa 3x1 para Migrantes, para el ejercicio fiscal 2014.

SEDESOL (2014). Acuerdo por el que se emiten las Reglas de Operación del Programa 3x1 para Migrantes, para el ejercicio fiscal 2015.

SEDESOL (2015). Acuerdo por el que se emiten las Reglas de Operación del Programa 3x1 para Migrantes, para el ejercicio fiscal 2016.

SEDESOL (2016). Acuerdo por el que se emiten las Reglas de Operación del Programa 3x1 para Migrantes, para el ejercicio fiscal 2017.

SEDESOL (2017). Acuerdo por el que se emiten las Reglas de Operación del Programa 3x1 para Migrantes, para el ejercicio fiscal 2018.

SERPRO (2005). Evaluación Externa del Programa Iniciativa Ciudadana 3x1, 2004.

SERPRO (2006). Evaluación Externa del Programa 3x1 para Migrantes, 2005.

Shannon, A. (2006). Las organizaciones transnacionales como agentes del desarrollo local. Retos y oportunidades del Programa 3 x 1 para Migrantes. En Fernández de Castro, R., García Zamora, R., y Vila Freyer, A., editores, El Programa $3 x 1$ para migrantes: ¿Primera política transnacional en México?, pp. 85-97. LX Legislatura, Miguel Ángel Porrúa, ITAM, UAZ, México D.F.

Strauss, A. L. y Corbin, J. (2002). Bases de la investigación cualitativa: técnicas y procedimientos para desarrollar la teoría fundamentada. Universidad de Antioquia Medellín, Medellín.

Universidad Autónoma de Chapingo (2006). Evaluación Externa del Programa 3x1 para Migrantes 2006 .

Valenzuela, B. (2007). La instauración del 3x1 en Jalisco. El acomodo de los gobiernos locales a una política adoptada por el gobierno del estado. En Fernández de Castro, R., García Zamora, R., y Vila Freyer, A., editores, El Programa 3x1 para Migrantes: ¿Primera política transnacional en México?, pp. 139-156. LX Legislatura, ITAM, UAZ, Miguel Ángel Porrúa, México D.F.

Vega Briones, G. (2004). La formación de clubes del Estado de México. Mecanismos de organización de nuevas comunidades de migrantes mexicanos en los Estados Unidos. En Zárate Hoyos, G., editor, Remesas de mexicanos y centroamericanos en Estados Unidos. Problemas y perspectiva. El Colegio de la Frontera Norte.
Vega Godínez, A. y Brindis Almazán, L. (2011). La reconfiguración de la imagen pública del migrante a través de las organizaciones migrantes y las remesas colectivas. Psicoperspectivas, 10(1):4668. 